\title{
NOTE ON POWER SERIES
}

MAX A. ZORN

1. The problem. The following question was raised by Bochner. Let $\sum \alpha_{i k} \xi^{i} \eta^{k}$ be a power series with complex coefficients, such that substitution of convergent power series $\sum_{1}^{\infty} \alpha_{i} \zeta^{i}$ and $\sum_{1}^{\infty} \beta_{i} \zeta^{i}$ for $\xi$ and $\eta$ produces always a convergent power series in $\zeta$. Is the double series $\sum \alpha_{i k} \xi^{i} \eta^{k}$ convergent?

The answer is yes; we present a proof which presupposes from function theory only the Cauchy estimate for the coefficients of polynomials in a complex variable:

$$
\left|\gamma_{k} \zeta_{0}^{k}\right| \leqq\left(|\zeta|=\left|\zeta_{0}\right|\right) \sup \left|\sum \gamma_{k} \zeta^{k}\right| .
$$

We note that this estimate is also valid in certain types of fields with non-Archimedian valuations, namely, those for which the values are dense and the index is infinite; this was shown by Schoebe in [1]. ${ }^{1}$

2. Homogeneous polynomials. We denote a vector $(\xi, \eta)$ by $x$ and introduce as the norm $\|x\|$ of $x$ the maximum of $|\xi|$ and $|\eta|$. A complex Banach space results which, as a complete metric space, is of the second category with respect to itself. We then consider homogeneous polynomials $P(x)=\sum_{i+k=n} \alpha_{i k} \xi^{i} \eta^{k}$; it is clear that $P(\zeta x)=\zeta^{n} P(x)$, that $P\left(x+\zeta x_{0}\right)$ is a polynomial in $\zeta$, and that $P$ is a continuous function of $x$.

The following three lemmata are immediate consequences of the estimate (C).

(2.1) Lemma. If $|P(x)| \leqq M$ for $\|x\| \leqq \zeta$, then $\left|\alpha_{i k} \xi^{i} \eta^{k}\right| \leqq M$ for $|\xi|,|\eta| \leqq \zeta$.

(2.2) Lemma. $|P(x)| \leqq(|\zeta|=1)$ sup $\left|P\left(x+\zeta x_{0}\right)\right|$.

This special case of the principle of the maximum is a special case of (C), applied to the constant term of $P\left(x+\zeta x_{0}\right)$, considered as a polynomial in $\zeta$. It is used in the proof of (2.3). $\|x\| \leqq \zeta$.

(2.3) Lemma. If $|P(x)| \leqq M$ for $\left\|x-x_{0}\right\| \leqq \zeta$, then $|P(x)| \leqq M$ for

Proof (compare [2, p. 590]): $|P(x)| \leqq(|\zeta|=1)$ sup $\left|P\left(\zeta x_{0}+x\right)\right|$ $=(|\zeta|=1)$ sup $\left|P\left(x_{0}+\zeta^{-1} x\right)\right| \leqq\left(\left\|x_{1}-x_{0}\right\| \leqq\|x\|\right)$ sup $\left|P\left(x_{1}\right)\right|$.

Received by the editors February 24, 1947.

1 Numbers in brackets refer to the references cited at the end of the paper. 
3. Proof of the theorem. We may now dispose of the problem by proving a slightly stronger result.

(3.1) THEOREM. If the substitution $\xi=\alpha \zeta, \eta=\beta \zeta$ produces from $\sum \alpha_{i k} \xi^{i} \eta^{k}$ always a power series with a nonvanishing radius of convergence then the series $\sum\left|\alpha_{i k} \xi^{i} \eta^{k}\right|$ converges for sufficiently small $|\xi|$ and $|\eta|$.

Proof. The result of the substitution is, with $(\alpha, \beta)=a$,

$$
\sum_{n=0}^{\infty}\left(\sum_{i+k=n} \alpha_{i k} \alpha^{i} \beta^{k}\right) \zeta^{n}=\sum_{0}^{\infty} P_{n}(a) \zeta^{n} .
$$

Now let $\delta$ be a complex number ${ }^{2}$ for which $0<|\delta|<1$; there will exist, for every vector $a$, an integer $m$ such that $\sum P_{n}(a)\left(\delta^{m}\right)^{n}=\sum P_{n}\left(a \delta^{m}\right)$ converges. We say that the set $D$ of the vectors $x$ for which $\sum P_{n}(x)$ converges is of the second category. For every vector is in one of the sets $\delta^{-m} D$; if $D$ were of the first category, the sets $\delta^{-m} D$ and therefore the whole space would be of the same character.

By virtue of the continuity of the functions $P_{n}$ there will exist (compare [3, p. 19]) a sphere $\left\|x-x_{0}\right\| \leqq \rho, \rho>0$, and an $M$ such that $\left|P_{n}(x)\right| \leqq M$ holds in it for all $n$. By Lemma (2.3), the same inequality is valid for $\|x\| \leqq \rho$; therefore, $\left|P_{n}(x)\right| \leqq M / 2^{n}$ will be true ${ }^{3}$ for $\|x\| \leqq \rho / 2$. By Lemma (2.1) we have, for $|\xi|,|\eta| \leqq \rho / 2$, the inequalities

$$
\left|\alpha_{i k} \xi^{i} \eta^{k}\right| \leqq M / 2^{n}=M / 2^{i+k},
$$

which establish the absolute convergence of the double series.

4. Comments. The main point of our arrangement was the weakening of the premise; this procedure was possible essentially because we worked with the complex numbers. It would be interesting to know whether the original conjecture holds in the real case.

\section{REFERENCES}

1. W. Schoebe, Beitraege zur Funktionentheorie in nichtarchimedisch bewerteten Koerpern, Halle, 1930.

2. M. A. Zorn, Characterization of analytic functions in Banach spaces, Ann. of Math. vol. 46 (1945).

3. S. Banach, Thérie des opérations linéaires, Warsaw, 1932.

INDIANA UNIVERSITY

\footnotetext{
2 The introduction of the powers of a number in the place of the integers is a concession to the non-Archimedean case and otherwise not relevant.

${ }^{3}$ This inference can also be made in the non-Archimedean case, provided the valuation is dense.
} 